\title{
Characterization of Candida species from Intensive Care Unit Isolates in a Tertiary Care Centre in North-East India: A retrospective study
}

\section{Vikramjeet Dutta, Wihiwot Valarie Lyngdoh, Ishani Bora, \\ Basabdatta Choudhury, Annie Bakorlin Khyriem, Prithwis Bhattacharyya ${ }^{1}$ \\ Departments of Microbiology and ${ }^{1}$ Anaesthesiology, NEIGRIHMS, Mawdiangdiang, Shillong, Meghalaya, India}

Address for the Correspondence: Dr. Wihiwot Valarie Lyngdoh, Department of Microbiology, NEIGRIHMS, Mawdiangdiang, Shillong - 793 018,

Meghalaya, India

E-mail: drwvalarielyngdoh@ gmail.com

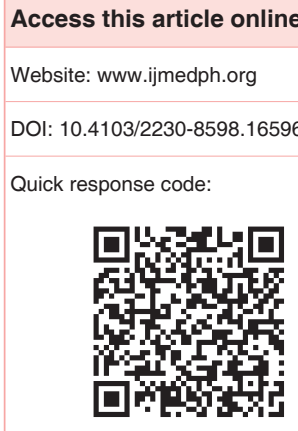

Background: Fungi have emerged as major causes of human diseases. Intensive Care Units (ICU), harbor almost all the risk factors for opportunistic fungal infections. Among these, Candida infections are very common with recent trends being rise in the non-Candida albicans (NCA) species along with an increase in resistance of these species to antifungal drugs. Aims: To characterize the Candida species from the clinical specimens of patients admitted in the ICU of Tertiary Care Centre in North-East India and to perform their antifungal susceptibility. Settings and Design: This retrospective study was conducted in the Department of Microbiology from January 2011 to December 2011. Materials and Methods: The following techniques were employed to characterize the isolates in the study - KOH mount, Gram's stain, India ink preparation, culture on Sabouraud's Dextrose Agar, Germ Tube test, Urea hydrolysis, morphology in Cornmeal Agar and chromogenic agar media, sugar fermentation and sugar assimilation tests and automated identification system, and the results were interpreted using standard protocols. Statistical Analysis Used: SPSS version 17.0 was used for all statistical computations and $P<0.05$ was taken as significant. Results: Out of 85 Candida isolates, Candida tropicalis (38\%) was the most common, in all age groups. Infections were more common in patients above 40 years and males were affected more than females. NCA species were more resistant to fluconazole than $C$. albicans. Conclusions: The study highlights the change in epidemiology in the species distribution of Candida and a rise in infections by NCA species as compared to those by $C$. albicans. Knowledge of the local species distribution of Candida along with their antifungal susceptibility is essential to initiate and optimize therapy and outcome, especially in an ICU setup, which harbors patients susceptible to fungal infections.

Key words: Antifungal susceptibility, Candida species, Intensive Care Unit, nonCandida albicans species, North-East India

\section{INTRODUCTION}

Fungi have emerged as major causes of human diseases and fungal infections have led to important public health problems since the early 1980s. Especially, among the immunocompromised and those hospitalized with serious underlying diseases, opportunistic fungal infections bring about a substantial increase in morbidity and mortality. ${ }^{[1]}$ Among the various risk factors for developing

This is an open access article distributed under the terms of the Creative Commons AttributionNonCommercial-ShareAlike 3.0 License, which allows others to remix, tweak, and build upon the work non-commercially, as long as the author is credited and the new creations are licensed under the identical terms.

For reprints contact: reprints@medknow.com

How to cite this article: Dutta V, Lyngdoh WV, Bora I, Choudhury B, Khyriem AB, Bhattacharyya P. Characterization of Candida species from Intensive Care Unit Isolates in a Tertiary Care Centre in North-East India: A retrospective study. Int J Med Public Health 2015;5:312-6. 
fungal infections, the most important ones are an ever-expanding population with immuno-compromised states due to mucosal or cutaneous barrier disruption, defects in the number and function of neutrophils or in cell-mediated immunity, metabolic dysfunction, and extremes of age. Other risk factors include sepsis, different surgical procedures, increasing use of broad-spectrum antibiotics, cytotoxic chemotherapies, transplantations, parenteral nutrition, multiple-lumen catheter use, prior Candida species colonization, renal replacement therapy, mechanical ventilation, etc., which often involve the collateral damage of circumventing the body's normal defense mechanisms thus further increasing the risk for both common and uncommon opportunistic fungal infections. ${ }^{[2]}$ Intensive Care Units (ICU), which though typically represent only about $5 \%$ of hospital beds, but are host to more than $20 \%$ of hospital infections and harbor almost all the risk factors for opportunistic fungal infections, provide such a suitable environment. ${ }^{[3]}$

Among the formidable list of opportunistic fungi, without question the single most important cause of opportunistic mycoses worldwide remains Candida. Candida species are responsible for up to $78 \%$ of nosocomial fungal infections. Overall, they are the sixth most common nosocomial pathogen, and fourth most common microorganism in nosocomial sepsis cases according to the National Nosocomial Infection Surveillance system. ${ }^{[4]}$ Although worldwide increase in the incidence of invasive Candida infections has been witnessed since the 1980s, the recent trends demonstrate a gradual change in its species distribution, with many countries experiencing a relative rise in the proportion of non-Candida albicans (NCA) isolates. Moreover though C. albicans is generally susceptible to fluconazole, decreased in vitro susceptibility to fluconazole is more common among several NCA species; however, the clinical relevance of this fact is not well defined. ${ }^{[5,6]}$

India has a high prevalence of invasive candidiasis owing to the presence of a number of contributory factors including favorable climatic conditions, a large population of immuno-compromised hosts including people living with HIV/AIDS and diabetes mellitus, and widespread access to antibiotics and steroids without prescription. ${ }^{[7]}$ Candida species is ranked fifth among nosocomial urinary pathogens and is also the eighth most common blood stream pathogen in India. ${ }^{[8,9]}$ Despite the availability of a few studies from the North-East India, ${ }^{[10]}$ lack of adequate diagnostic mycology laboratory procedures, precludes the availability of representative data on the epidemiological and mycological characteristics of invasive candidiasis occurring in vast stretches of the country. Nevertheless, because of the immense eco-geographical heterogeneity in the country and in view of the geographical and temporal variation often observed in the species distribution of Candida,${ }^{[5]}$ there is a need to investigate and monitor local epidemiological patterns of Candida infections in India.

The increasing population of immuno-compromised patients, together with the rising incidence of NCA species and the emergence of acquired antifungal resistance, necessitates the judicious administration of antifungal prophylaxis in at-risk patients and empirical antifungal therapy in patients suffering from candidiasis. Characterization and sensitivity profiles of the locally prevalent Candida strains and knowledge regarding risk factors relevant for the patient profile are utmost essential for the judicious administration of antifungal prophylaxis as well as antifungal therapy.

Hence, the current study was undertaken with the aim to characterize the Candida species obtained from the clinical specimens of patients admitted in the ICU of our hospital and to perform their antifungal susceptibility.

\section{MATERIALS AND METHODS}

A retrospective study was undertaken with the Candida isolates obtained from clinical specimens of patients admitted in the ICU for a period of 1-year from January 2011 to December 2011, in the Department of Microbiology in a tertiary care set-up in North-East India. It has a 32 bedded ICU catering to various patient profiles. Candida isolated from the samples collected from ICU were subjected to speciation using the following techniques according to standard protocols ${ }^{[11,12]}-\mathrm{KOH}$ mount, Gram's stain, India Ink preparation, culture on Sabouraud's Dextrose Agar, Germ Tube test, Urea hydrolysis, Cornmeal Agar (CMA) morphology (Dalmau technique), morphology in chromogenic agar media, Sugar fermentation test, Sugar assimilation test (Auxanographic Plate Method) and then finally subjected to automated identification system (Vitek 2 Compact, Biomerieux), using protocols prescribed by the manufacturer. Sugar assimilation test was taken as the gold standard for identification of the Candida species. The patient profile and associated risk factors were noted. The antifungal susceptibility was done according to Clinical Laboratory Standards Institute (CLSI) guideline, method for antifungal disc diffusion susceptibility for yeasts, with approved guideline M44-A. ${ }^{[13]}$ We compared the sensitivity profile of the organisms to two antifungals - fluconazole $(25 \mu \mathrm{g})$ and amphotericin B $(10 \mu \mathrm{g})$. The inoculum was standardized to $0.5 \mathrm{McFarland}$ units. Standard ATCC strains (C. albicans, ATCC 90028, Candida parapsilosis, ATCC 22019 and Candida krusei, ATCC 6258) were used as a control.

\section{Statistical analysis}

Differences in antifungal sensitivity between C. albicans and NCA species were also examined for statistical significance using Fisher's exact test. SPSS version 17.0 (SPSS Inc. Released 2008. SPSS Statistics for Windows, Version 17.0. Chicago: SPSS Inc.) was used for all statistical computations and $P<0.05$ was taken as significant.

\section{RESULTS}

A total of 266 samples from 237 patients were received, from the ICU of our hospital during the study period. Of these, a total of 85 Candida isolates were obtained from 74 patients.

Among the various co-morbid conditions observed in the patients, cerebro-vascular accidents $(23 \%)$ was the most common, followed 
by tuberculosis $(15 \%)$, respiratory distress $(12 \%)$, patients in postoperative state $(11 \%)$ and others [Table 1].

In this study, among the 85 Candida species isolated, NCA species were more in number than $C$. albicans. The most common among NCA species included Candida tropicalis (38\%), Candida lusitaniae (13\%), Candida glabrata (9\%), Candida parapsilosis (7\%), Candida krusei $(6 \%)$ and Candida guilliermondii (4\%). A single isolate each of Candida kefyr and Candida zeylanoides was also obtained. C. albicans accounted for $9 \%$ of the isolates. About $12 \%$ of the Candida species isolated could not be identified conclusively by any of the identification methods.

The highest number of samples were received in the age group of $41-60$ years $(49 \%)$, followed by the age groups of $>60$ years $(27 \%)$, $21-40$ years $(15 \%)$ and $<20$ years $(9 \%)$. Maximum isolates obtained were also from the age group of 41-60 years (45\%), followed by the age groups of $>60$ years $(26 \%), 21-40$ years $(18 \%)$ and $<20$ years $(11 \%)$.

C. tropicalis was the most common isolate in all age groups. In the age group 41-60 years in which the maximum isolates were obtained, C. tropicalis (34\%) was the predominant isolate, followed by C. lusitaniae (13\%), C. glabrata (1\%), C. albicans and C. krusei (8\%, each), C. parapsilosis and C. guilliermondii (5\%, each). The single isolates of $C$. kefyr and C. zeylanoides were obtained in the age groups of 41-60 years and $>60$ years, respectively.

Candida species were isolated more in males (62\%) than females in the present study. C. tropicalis, the most isolated species, was also more common in the males $(72 \%)$ than females [Table 2].

Candida species were isolated mainly from urine samples $(37 \%)$, followed by sputum and Endo-tracheal secretions (21\%), throat swab (16\%), Ryle's tube aspirate $(12 \%)$, pus and exudates $(8 \%)$, blood (4\%) and cerebrospinal fluid (CSF; 2\%) [Table 3].

Out of 85 samples, 55 samples showed consistent identification results by the entire range of diagnostic tests used but 30 samples exhibited discordant results on CMA and Vitek 2 Compact tests. The result of the sugar assimilation test was considered as the confirmatory finding for these 30 samples. 18 samples identified by Vitek 2 Compact showed concordance with sugar assimilation test; 23 samples identified by CMA showed concordance with sugar assimilation test. The difference was however not statistically significant $(P=0.27)$. The Candida isolates were also cultured in chromogenic agar media for identification.

\begin{tabular}{lc} 
Table 1: Co-morbid conditions associated with \\
Candida infections in ICU \\
\hline Co-morbid conditions & Number of patients \\
\hline Respiratory distress & 9 \\
Acute renal failure (with/without sepsis) & 3 \\
Tuberculosis (pulmonary/extra pulmonary) & 11 \\
Diabetes mellitus (with complications) & 5 \\
Cerebro-vascular accident & 17 \\
Multiple infarct & 3 \\
Chronic obstructive pulmonary disease & 7 \\
Cerebral malaria & 6 \\
Alcoholic liver disease/hepatic encephalopathy & 2 \\
Postoperative state & 8 \\
Seizures & 3 \\
Respiratory distress & 9 \\
Total & 74 \\
\hline
\end{tabular}

$\mathrm{ICU}=$ Intensive care unit

\begin{tabular}{|c|c|c|c|c|c|}
\hline \multirow[t]{2}{*}{ Candida isolates } & \multicolumn{4}{|c|}{ Age-groups (in years) } & \multirow{2}{*}{$\begin{array}{c}\text { Total } \\
\text { (male/female) }\end{array}$} \\
\hline & $<20$ & $21-40$ & $41-60$ & $>60$ & \\
\hline Candida albicans & 1 & 1 & 3 & 3 & $8(5 / 3)$ \\
\hline Candida tropicalis & 3 & 7 & 13 & 9 & $32(23 / 9)$ \\
\hline Candida krusei & 0 & 1 & 3 & 1 & $5(3 / 2)$ \\
\hline Candida parapsilosis & 1 & 1 & 2 & 2 & $6(3 / 3)$ \\
\hline Candida Iusitaniae & 2 & 2 & 5 & 2 & $11(7 / 4)$ \\
\hline Candida glabrata & 1 & 2 & 4 & 1 & $8(6 / 2)$ \\
\hline Candida guilliermondii & 0 & 0 & 2 & 1 & $3(1 / 2)$ \\
\hline Candida kefyr & 0 & 0 & 1 & 0 & $1(0 / 1)$ \\
\hline Candida zeylanoides & 0 & 0 & 0 & 1 & $1(1 / 0)$ \\
\hline Unidentified & 2 & 1 & 5 & 2 & $10(4 / 6)$ \\
\hline Total & 10 & 15 & 38 & 22 & $85(53 / 32)$ \\
\hline
\end{tabular}

$\mathrm{ICU}=$ Intensive care unit

\begin{tabular}{|c|c|c|c|c|c|c|c|c|}
\hline Candida isolates & Urine & $\begin{array}{c}\text { Sputum/Endo- } \\
\text { tracheal secretion }\end{array}$ & Blood & $\begin{array}{l}\text { Throat } \\
\text { swab }\end{array}$ & $\begin{array}{l}\text { Ryle's tube } \\
\text { aspirate }\end{array}$ & CSF & $\begin{array}{c}\text { Pus/ } \\
\text { exudate }\end{array}$ & Total \\
\hline Candida albicans & 1 & 3 & 0 & 2 & 2 & 0 & 0 & 8 \\
\hline Candida tropicalis & 11 & 7 & 1 & 6 & 4 & 1 & 2 & 32 \\
\hline Candida krusei & 2 & 1 & 0 & 1 & 1 & 0 & 0 & 5 \\
\hline Candida parapsilosis & 3 & 1 & 0 & 1 & 0 & 0 & 1 & 6 \\
\hline Candida Iusitaniae & 6 & 2 & 1 & 0 & 1 & 0 & 1 & 11 \\
\hline Candida glabrata & 5 & 1 & 0 & 1 & 1 & 0 & 0 & 8 \\
\hline Candida gullerimondi & 0 & 1 & 0 & 1 & 0 & 0 & 1 & 3 \\
\hline Candida kefyr & 0 & 0 & 0 & 0 & 0 & 0 & 1 & 1 \\
\hline Candida zeylanoides & 0 & 0 & 0 & 1 & 0 & 0 & 0 & 1 \\
\hline Unidentified & 3 & 2 & 1 & 1 & 1 & 1 & 1 & 10 \\
\hline Total & 31 & 18 & 3 & 14 & 10 & 2 & 7 & 85 \\
\hline
\end{tabular}

CSF = Cerebrospinal fluid, ICU = Intensive care unit 
In this study, none of the isolates showed resistance to amphotericin B $(10 \mu \mathrm{g})$. As far as resistance to fluconazole $(25 \mu \mathrm{g})$ is concerned, C. glabrata showed highest resistance $(72 \%)$ followed by C. tropicalis (43\%), C. parapsilosis (37\%), C. albicans (23\%) and C. lusitaniae (13\%). The difference in resistance of NCA species and $C$. albicans to fluconazole was found to be statistically significant $(P=0.0087)$ [Figure 1].

\section{DISCUSSION}

Candida species are the most common agents of fungal infection in humans affecting skin, nails, mucosa and internal organs of the body. ${ }^{[14]}$ The species are endogenous in nature and are usually responsible for opportunistic infections.

In the present study, we observed that NCA species were more frequently encountered than $C$. albicans. In different studies around the globe ${ }^{[5,15-18]}$ and in India, ${ }^{[6,9,19-23]}$ it was observed that, NCA species were predominant (30-90\%) among the isolated Candida species. Some authors have attributed this emergence of NCA species to the prophylactic use of fluconazole, in the species distribution of Candida, but it is not the factor in our study. Various co-morbid conditions of the patients, stay in ICU, and use of invasive monitoring devices and administration of broad-spectrum antibiotics as combination therapy were a few notable risk factors that could have led to the current spectrum of NCA species prevalence in the present study. Further studies with larger sample size are needed to verify our findings.

C. tropicalis was the most common among NCA species in this study. The isolation of more NCA species as compared to C. albicans is in agreement with various other studies on Candida infection. ${ }^{[23-28]}$

In the present study, males were more affected than females with an overall male:female ratio of 1.55 . The preponderance of male patients suffering from candidiasis in this study correlates with those of Kashid et al. and Singh et al. ${ }^{[23,29]}$ Infections were more common in the older age groups in our study. This can be attributed to the various co-morbid conditions and the health issues pertaining to the particular age-groups, as is relevant in our study. However, further studies have to be carried out to justify the significance of the fact.

The disk diffusion method, according to CLSI guidelines, was used for antifungal susceptibility testing as it is simple and easy to perform, but it is only a preliminary screening procedure. To confirm resistance of any strain, broth dilution tests should be used to determine the minimum inhibitory concentrations (MIC) of the drugs. The isolates in the study showed no resistance to amphotericin B. The NCA species showed more resistance than C. albicans to fluconazole and this finding was found to be statistically significant $(P=0.0087)$. These findings corelates with studies by Binesh and Kalyani and Kashid et al..$^{[22,23]}$

Our study is limited by a retrospective design, relatively smaller sample size, a lesser group of antifungals tested, a lack of

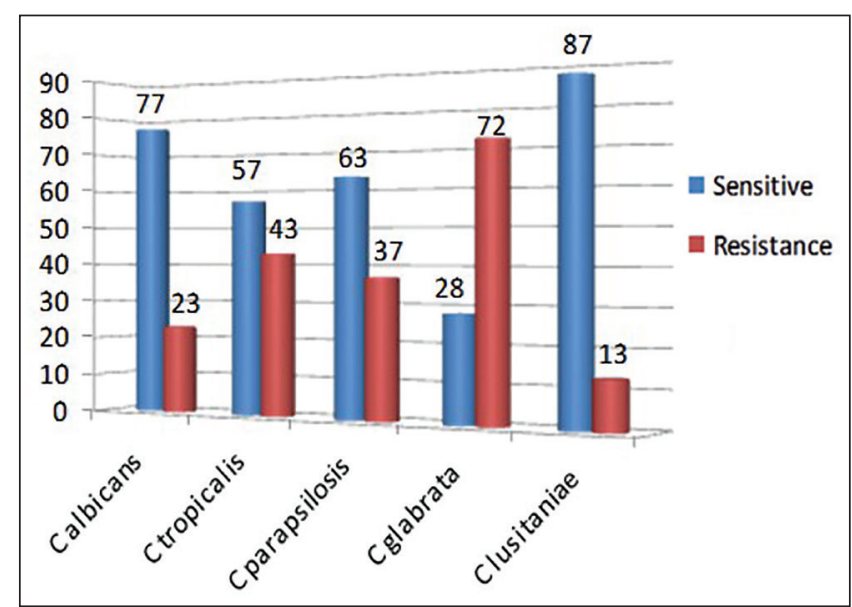

Figure 1: Susceptibility pattern of Candida species to fluconazole, $25 \mu \mathrm{g}(\%)$

information regarding the MIC values of the antifungals tested and clinical outcome of the patients. The isolation of a smaller numbers of some of the NCA species like C. krusei, C. gullerimondii, C. kefyr, C. zeylanoides etc., precludes our ability to evaluate the clinical significance of these species, although an overall increase in resistance was significantly observed in the NCA species. Therefore, further studies with a larger sample size, elaborate clinical data with treatment details and outcome of the patients and testing of different groups of antifungals along with their MIC values are required for a better understanding of the sure and steady rise of fungal infections, and especially by the NCA species.

The present study highlights the change in epidemiology in the species distribution of Candida and also highlights a rise in the infections by NCA species as compared to those by C. albicans, along with an increase in resistance of these NCA species to the routinely used antifungals. Therefore knowledge of the local species distribution of Candida through presumptive identification, followed by confirmation, is essential to initiate early empirical therapy, especially in an ICU setup, which harbors a lot of immunocompromised patients and other patients susceptible for acquiring various fungal infections. Moreover, antifungal susceptibility testing, though not as routinely performed as antibacterial susceptibility testing, should be carried out, along with MIC values of the isolates for the various groups of antifungals, in order to optimize therapy and outcome.

\section{Financial support and sponsorship}

Nil.

\section{Conflicts of interest}

There are no conflicts of interest.

\section{REFERENCES}

1. Pfaller MA, Diekema DJ. Epidemiology of invasive candidiasis: A persistent public health problem. Clin Microbiol Rev 2007;20:133-63.

2. Zaragoza R, Pemán J. The diagnostic and therapeutic approach to fungal 
infections in critical care settings. Adv Sepsis 2008;6:90-8.

3. Pittet D, Harbarth SJ. The intensive care unit. In: Bennett JV, Brachman PS, editors. Hospital Infections. $4^{\text {th }}$ ed. Pennsylvania: Lippincott-Raven Press; 1998. p. 381-402.

4. Beck-Sagué $C$, Jarvis WR. Secular trends in the epidemiology of nosocomial fungal infections in the United States, 1980-1990. National Nosocomial Infections Surveillance System. J Infect Dis 1993; 167:1247-51.

5. Pfaller MA, Diekema DJ; International Fungal Surveillance Participant Group. Twelve years of fluconazole in clinical practice: Global trends in species distribution and fluconazole susceptibility of bloodstream isolates of Candida. Clin Microbiol Infect 2004;10 Suppl 1:11-23.

6. Shivaprakasha S, Radhakrishnan K, Karim PM. Candida spp. other than Candida albicans: A major cause of fungaemia in a tertiary care centre. Indian J Med Microbiol 2007;25:405-7.

7. Chakrabarti A, Chatterjee SS, Shivaprakash MR. Overview of opportunistic fungal infections in India. Nihon Ishinkin Gakkai Zasshi 2008;49:165-72.

8. Prasad KN, Agarwal J, Dixit AK, Tiwari DP, Dhole TN, Ayyagari A. Role of yeasts as nosocomial pathogens \& their susceptibility to fluconazole \& amphotericin B. Indian J Med Res 1999;110:11-7.

9. Verma AK, Prasad KN, Singh M, Dixit AK, Ayyagari A. Candidaemia in patients of a tertiary health care hospital from North India. Indian J Med Res 2003;117:122-8.

10. Eicher AD, Crofts N, Benjamin S, Deutschmann P, Rodger AJ. A certain fate: Spread of HIV among young injecting drug users in Manipur, North-East India. AIDS Care 2000;12:497-504.

11. Chander J. Textbook of Medical Mycology. $3^{\text {rd }}$ ed. New Delhi: Metha Publishers; 2010.

12. Milne LJ. Fungi. In: Collee JG, Fraser AG, Marmion BP, Simmons A, editors. Mackie \& McCartney Practical Medical Microbiology. $14^{\text {th }}$ ed. Churchill Livingstone, Elsevier (Singapore) Pte. Ltd.; 1996. p. 695-717.

13. Clinical and Laboratory Standards Institute/National Committee for Clinical Laboratory Standards. Method for Antifungal Disk Diffusion Susceptibility Testing of Yeasts: Approved Guideline. Document M44-A. Wayne, PA: Clinical and Laboratory Standards Institute; 2004.

14. Vazquez JA, Sobel JD. Mucosal candidiasis. Infect Dis Clin North Am 2002;16:793-820, v.

15. Vincent JL, Rello J, Marshall J, Silva E, Anzueto A, Martin CD, et al. International study of the prevalence and outcomes of infection in intensive care units. JAMA 2009;302:2323-9.
16. Méan M, Marchetti $\mathrm{O}$, Calandra T. Bench-to-bedside review: Candida infections in the intensive care unit. Crit Care 2008;12:204.

17. El-Mashad N, Mahmoud MT. Commercial tests for in vitro antifungal susceptibility testing of Candida species compared to standard (NCCLS) broth microdilution. Int J Med Med Sci 2011;3:100-4.

18. Pfaller MA. Antifungal drug resistance: Mechanisms, epidemiology, and consequences for treatment. Am J Med 2012;125 (1 Suppl):S3-13.

19. Vijaya D, Malini. Characterization and antifungal susceptibility of Candida species: A preliminary study. J Acad Clin Microbiol 2000; 2:55-7.

20. Rani R, Mohapatra NP, Mehta G, Randhawa VS. Changing trends of Candida species in neonatal septicaemia in a tertiary North Indian hospital. Indian J Med Microbiol 2002;20:42-4

21. Capoor MR, Nair D, Deb M, Verma PK, Srivastava L, Aggarwal P. Emergence of non-albicans Candida species and antifungal resistance in a tertiary care hospital. Jpn J Infect Dis 2005;58:344-8.

22. Binesh LY, Kalyani M. Phenotypic characterization of Candida species and their antifungal susceptibility from a tertiary care centre. J Pharm Biomed Sci 2011;11:1-4.

23. Kashid RA, Belawadi S, Devi G, Indumati. Characterisation and antifungal susceptibilty testing for Candida species in a tertiary care hospital. J Health Sci Res 2011;2:1-7.

24. Chakrabarti A, Mohan B, Shrivastava SK, Marak RS, Ghosh A, Ray P. Change in distribution \& antifungal susceptibility of Candida species isolated from candidaemia cases in a tertiary care centre during 19962000. Indian J Med Res 2002;116:5-12.

25. Agarwal J, Bansal S, Malik GK, Jain A. Trends in neonatal septicemia: Emergence of non-albicans Candida. Indian Pediatr 2004;41:712-5.

26. Singhi SC, Reddy TC, Chakrabarti A. Oral itraconazole in treatment of candidemia in a pediatric intensive care unit. Indian $\mathrm{J}$ Pediatr 2004;71:973-7.

27. Agarwal S, Manchanda V, Verma N, Bhalla P. Yeast identification in routine clinical microbiology laboratory and its clinical relevance. Indian J Med Microbiol 2011;29:172-7.

28. Jain N, Mathur P, Misra MC, Behera B, Xess I, Sharma SP. Rapid identification of yeast isolates from clinical specimens in critically ill trauma ICU patients. J Lab Physicians 2012;4:30-4.

29. Singh K, Chakrabarti A, Narang A, Gopalan S. Yeast colonisation \& fungaemia in preterm neonates in a tertiary care centre. Indian $\mathrm{J}$ Med Res 1999;110:169-73. 